\title{
NOTE ON A WHITEHEAD PRODUCT
}

GADE V. KRISHNARAO

The aim of this note is to prove

THEOREM. Let $\iota_{n}$ generate $\pi_{n}\left(S^{n}\right)$ and $n=4 s+1, s$ being a positive integer. Then $\left[\iota_{n}, \iota_{n}\right]$ is not divisible by two.

Let $i, j$, and $k$ be homomorphisms in the exact sequences of the fiber bundles $O(n) \rightarrow O(n+1) \rightarrow S^{n}, n \geqq 1$

$$
\cdots \pi_{m}(O(n)) \stackrel{i}{\rightarrow} \pi_{m}(O(n+1)) \stackrel{j}{\rightarrow} \pi_{m}\left(S^{n}\right) \stackrel{k}{\rightarrow} \pi_{m-1}(O(n))
$$

and $d=j \circ k$ be the composite

$$
\pi_{m+1}\left(S^{n+1}\right) \stackrel{k}{\rightarrow} \pi_{m}(O(n+1)) \stackrel{j}{\rightarrow} \pi_{m}\left(S^{n}\right) .
$$

We state an easy lemma without proof.

LemMA 1. Let $(E, F) \rightarrow\left(B,{ }^{*}\right)$ be a fibration and $\partial$ be the boundary homomorphism in its exact homotopy sequence. Then $\partial(\alpha \circ E \beta)=\partial(\alpha) \circ \beta$ where $\alpha \in \pi_{r}(B), E \beta \in \pi_{q}\left(S^{r}\right)$ and $E$ is the suspension homomorphism.

Lemma 2. For odd $n,\left[\iota_{n}, \iota_{n}\right]$ is divisible by two if and only if it is in the image of $d$.

Proof. It is known [3, p. 120] that $d\left(\iota_{n+1}\right)=2 \iota_{n}$ if $n$ is odd. Since $E: \pi_{2 n-1}\left(S^{n}\right) \rightarrow \pi_{2 n}\left(S^{n+1}\right)$ is onto and its kernel is generated by $\left[\iota_{n}, \iota_{n}\right]$ and also $E: \pi_{2 n-2}\left(S^{n-1}\right) \rightarrow \pi_{2 n-1}\left(S^{n}\right)$ is onto, we have, for any element $E \alpha$ in $\pi_{2 n}\left(S^{n+1}\right)$

$$
\begin{aligned}
d(E \alpha) & =j \circ k(E \alpha)=j \circ k\left(\iota_{n+1} \circ E \alpha\right) \\
& =2 \iota_{n} \circ \alpha \quad \text { by Lemma } 1 \\
& =2 \alpha .
\end{aligned}
$$

If $\left[\iota_{n}, \iota_{n}\right]=2 \alpha$, for some $\alpha$ in $\pi_{2 n-1}\left(S^{n}\right)$, then $E \alpha \neq 0$ and $d(E \alpha)=2 \alpha$. That proves the lemma.

If $n=4 s+1, S^{n}$ admits only a 1-field. Consequently [2], (i) there exists $b \in \pi_{n-1}(O(n-1))$ such that $i(b)=k\left(\iota_{n}\right)$ and $j(b)=\eta$ where $\eta$ is the stable element in $\pi_{n-1}\left(S^{n-2}\right)$ (we denote all suspensions of $\eta$ by the same symbol), and (ii) $\left[\iota_{n}, \iota_{n}\right]=E \theta$ where $\theta \in \pi_{2 n-2}\left(S^{n-1}\right)$ is not a suspension.

Received by the editors December 13, 1965. 


$$
j \circ(i)^{-1} \circ k(E \theta)=\eta \circ \theta \quad \text { by Lemma } 1 .
$$

Consider the generalized Hopf invariant

$$
\begin{aligned}
& H: \pi_{2 n-2}\left(S^{n-2}\right) \rightarrow \pi_{2 n-2}\left(S^{2 n-5}\right), \\
& H(\eta \circ \theta)=E(\eta \# \eta) \circ H(\theta), \quad \text { see }[4, \text { p. 18] } \\
& =\eta \circ \eta \circ H(\theta), \quad \text { see [1] } \\
& =\eta \circ \eta \circ \eta \neq 0
\end{aligned}
$$

because $\theta$ is not a suspension, $H(\theta) \neq 0$ and hence $H(\theta)=\eta$. Therefore $\eta \circ \theta \neq 0$ and $k(E \theta) \neq 0$ and the exactness of (A) implies that $E \theta$ $=\left[\iota_{n}, \iota_{n}\right]$ is not in the image of $d=j \circ k$. This proves the Theorem.

\section{REFERENCES}

1. M. G. Barratt and P. J. Hilton, On join operations in homotopy groups, Proc. London Math. Soc. 53 (1953), 430-445.

2. I. M. James, Whitehead products and vector fields on spheres, Proc. Cambridge Philos. Soc. 53 (1957), 817-820.

3. N. Steenrod, The topolngy of fibre bundles, Princeton Mathematical Series, Vol. 14, Princeton Univ. Press, Princeton, N. J. 1951.

4. H. Toda, Composition methods in homotopy groups of spheres, Annals of Mathematics Studies, No. 49, Princeton Univ. Press, Princeton, N. J., 1962.

Indian Institute of Technology, Kanpur 